\title{
Correlation between Acoustic Intensity and Ground Particle Size in Alumina Ball Mill Process
}

\author{
Kyeong-Sik Cho*,, Soo-Hyun Kim*, and Young Hun Lee** \\ *School of Advanced Materials Science and Engineering, Kumoh National Institute of Technology, Gumi 39177, Korea \\ **School of Electrical Engineering, Kumoh National Institute of Technology, Gumi 39177, Korea
}

(Received February 7, 2018; Revised March 19, 2018; Accepted March 20, 2018)

\begin{abstract}
In the ball milling process of ceramic powders, according to economic considerations for industrial applications, it is very important to quickly determine the optimum process condition with the maximum grinding efficiency. However, it is still difficult to determine the optimum condition for a ball mill with respect to the various process parameters, such as the rotational speed and the milling time. Ball milling was carried out at the same starting conditions with given amounts of alumina powders, balls and water, and was conducted slower or faster or a critical rotational speed was just determined by observing the angular position of the slurry in a semi-translucent polyethylene laboratory container. With respect to the different rotational speeds, which were slower or faster than the critical rotational speed, the particle size distribution of the grained powders and the acoustic intensity caused by cascading of the balls led to various behaviors. From the results of the particle size distribution and the acoustic signal analysis in the ball milling, there was one rotational speed that made the finest milled powder with maximum acoustic intensity. As a result, there was a correlation between the ground particle size and the acoustic intensity, which yields the interpretation that it can be possible in-situ to determine the optimum condition of ball milling by acoustic signal without repeated measurement efforts.
\end{abstract}

Key words : Milling, Powders, $\mathrm{Al}_{2} \mathrm{O}_{3}$, Critical rotational speed, Acoustic intensity

\section{Introduction}

$I^{n}$ n order to manufacture ceramic materials of outstanding quality through a series of processes beginning with powder as the starting material, prerequisite conditions include the size of the powder material, which needs to be small; the particle size distribution, which needs to be narrow; and the powder particle shape, which has to be spherical. Ball milling is one treatment process that is widely used for the purpose of grinding and mixing powder materials in laboratories and sites of ceramic material fabrication. Ball milling is a method for grinding and mixing the powder raw material using friction and collision energy produced through the movement of the powder material to be grinded and balls placed together in a cylindrical jar and rotated. ${ }^{1)}$

In the ball milling process, the grinded powder particle size and shape are affected by numerous variables including the jar material, jar size, jar shape, number of balls and amount of powder material, and viscosity of the slurry, which is a mixture of the ingredient materials and depends on the utilized dispersion medium, the rotational speed, and the rotation time. ${ }^{1-5)}$ The materials of the jar and balls have to be of similar or equivalent composition with the ingredient ceramic powder in order to reduce the inclusion of impu-

\footnotetext{
${ }^{\dagger}$ Corresponding author : Kyeong-Sik Cho

E-mail : kscho@kumoh.ac.kr

Tel : +82-54-478-7737 Fax : +82-54-478-7769
}

rities; or, materials such as polyurethane and polyethylene are embedded in the jar because these materials can be removed in the calcination process afterwards. ${ }^{1)}$ When processing industrial ingredient powders of relatively small size, the grinding rate increases as the ball diameter decreases and the lower bound of the minimum size that can be achieved decreases. ${ }^{3)}$ The grinding rate is optimal when the balls fill the jar capacity to around $40 \sim 45 \%$; smaller or greater quantities can lead to inadequate grinding as smaller amounts result in abrasion of the jar and greater amounts result in decreased space for movement of the balls. ${ }^{3,5)}$ As the rotational speed increases, the grinding efficiency increases, ${ }^{6}$ ) and a lifter bar is embedded within the jar so that the internal contents can move to a higher position and fall, increasing the grinding efficiency even at low speeds. ${ }^{7}$ Since the powder amount in wet ball milling affects the slurry viscosity, the powder amount needs to be appropriate so as not to impact the friction and collision number of the balls; a low powder amount leads to decreased powder grinding capacity.

In order to realize grinded powder in accordance with the specifications, various process variables of the ball milling equipment need to be compiled and determined through particle size data analysis of the grinded powder according to the rotational speed and time. Thus, the determination of efficient grinding conditions for ball milling can be carried out through analysis of data accumulated from numerous experiments. While various formulas regarding the critical 
rotation speed have been proposed to minimize such experimentation, such formulas for optimal grinding were empirically obtained using a limited number of variables, and so significant deviations in the resulting grinded particle size were observed. ${ }^{2-4,8-10)}$

Also, the positions of internal content movement (powder and balls) during the ball milling process inside the jar have been studied previously. ${ }^{11)}$ When the rotational speed is slow, the internal contents shift towards the bottom rather than the center of the cylindrical jar, so that the balls rotate. At the critical rotational speed, the components slant towards the rotational direction at the bottom region, so that not only does rotational motion occur but also there are components that, due to centrifugal force, move up the internal jar wall and then fall. When the rotational speed is fast, the internal contents stick to the internal wall due to centrifugal force, resulting in a decreased grinding effect. Meanwhile, specific sounds can be heard due to vibration resulting from rotation of the mechanical equipment during ball milling, along with friction and collisions between the jar internal contents. It was reported in a previous study that the acoustic signal could contain information regarding the ball mill operation conditions. The acoustic characteristics were interpreted for parameters including the rotational speed, amount of powder, number of balls, and slurry viscosity. ${ }^{11-16)}$

In this study, commercially available alumina powder was

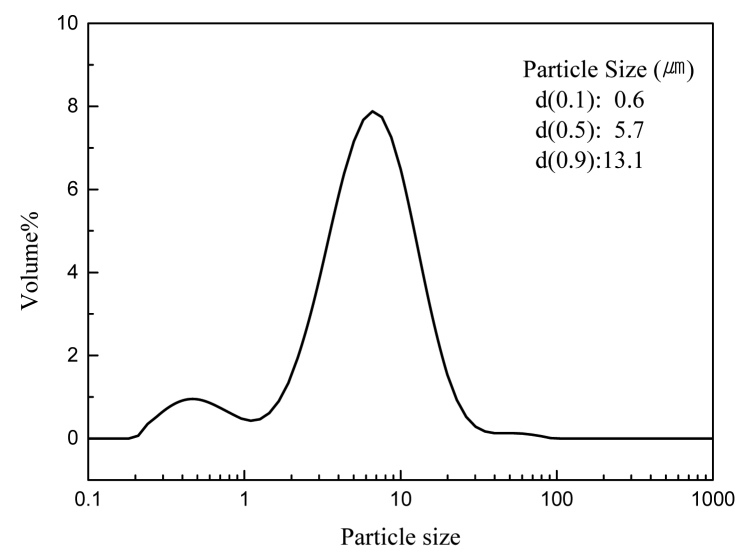

(a) used as the starting ingredient to conduct laboratory scale ball milling and analyze the series of particle sizes for the grinded powder, with the considered variables being the rotational speed and time. The acoustic signal obtained from the ball milling process was analyzed together with the process variables and the correlation between the grinded particle size and acoustic signal was extracted. Through this study, it is expected that the substantial amount of effort required to analyze the grinded particle size and determine the process parameters that represent the optimal grinding effect can be alleviated and the results produced right away through a simple on-site acoustic signal acquisition during the ball milling process.

\section{Experimental Procedure}

\subsection{Alumina Raw Material Powder and Ball Mill- ing Process Conditions}

The alumina powder used in this study to analyze the ball milling process was an industrial powder (CA-5M, Korea Alumina Co., Korea) with an average particle size of 6.92 $\mu \mathrm{m}$, purity of $99.6 \%$, normal particle size distribution, and form of irregular polyhedrons having rough surfaces (Fig. 1). Also, the ball milling used the slip casting process conditions widely used in laboratory scale, ${ }^{17-20)}$ and employed a $500 \mathrm{ml}$ semi-translucent polyethylene jar (Nalgene, Nalgene Labware, USA), spherical alumina balls with $3 \mathrm{~mm}$ diame-

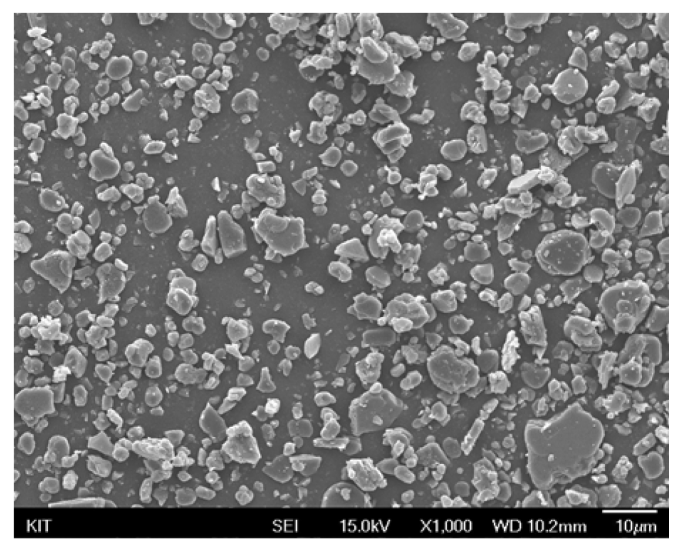

(b)

Fig. 1. Particle size distribution (a) and morphology (b) of the starting alumina powder.

Table 1. Ball Mill Operation Components for Alumina Powder

\begin{tabular}{|c|c|c|c|}
\hline Component & \multicolumn{2}{|c|}{ Specification } & Amount \\
\hline Alumina powder & \multicolumn{2}{|c|}{ CA-5M, Korea Alumina Co., Korea } & $200 \mathrm{~g}$ \\
\hline Milling jar & \multicolumn{2}{|c|}{ Polyethylene, Nalgene, Nalgene Labware, USA } & $500 \mathrm{ml}(\varnothing 7 \mathrm{~cm})$ \\
\hline Milling media & \multicolumn{2}{|c|}{ Alumina Ball (spherical $\varnothing 3 \mathrm{~mm}$ ) } & 50 vol $\%$ bottle filling \\
\hline \multirow{3}{*}{$\begin{array}{l}\text { Dispersion medium } \\
\text { and organic additive }\end{array}$} & \multicolumn{2}{|c|}{$\mathrm{H}_{2} \mathrm{O}$, D.I. water } & 60 vol\% water : 40 vol $\%$ powder \\
\hline & Dispersant & $\begin{array}{l}\left.\text { Ammonium polycarbonate, }\left(\mathrm{NH}_{4}\right)_{2} \mathrm{CO}_{3}\right) \mathrm{n}, 5468 \mathrm{CF}, 40 \% \\
\text { solution, San Nopco Korea Ltd., Korea }\end{array}$ & $0.8 \mathrm{~g}$ \\
\hline & Plasticizer & $\begin{array}{l}\text { Polyethylene glycol }\left(\mathrm{C}_{2} \mathrm{H}_{6} \mathrm{O}_{2}\right) \mathrm{n} \text {, PEG-400, Guoli Chem. } \\
\text { Co., Ltd., Korea }\end{array}$ & $2.0 \mathrm{~g}$ \\
\hline
\end{tabular}


ters, water as the dispersion medium, and other organic additives (Table 1).

To obtain the smallest grinded particle size, the optimal revolutionary speed in the ball milling process was determined by conducting the ball milling at rotational speeds ranging from $96 \sim 352 \mathrm{rpm}$ and observing the side of the semi-translucent jar for the spatial position distribution of the internal contents (powder and balls), moving according to the rotational speed. A bright light was shone on the upper left portion of the semi-translucent jar and videos

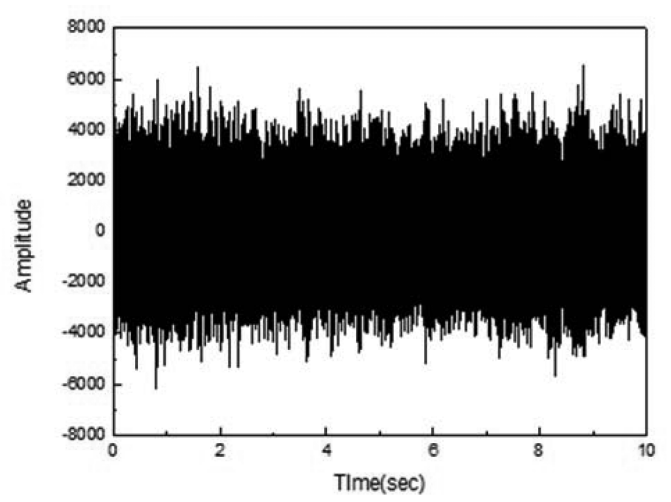

(a)

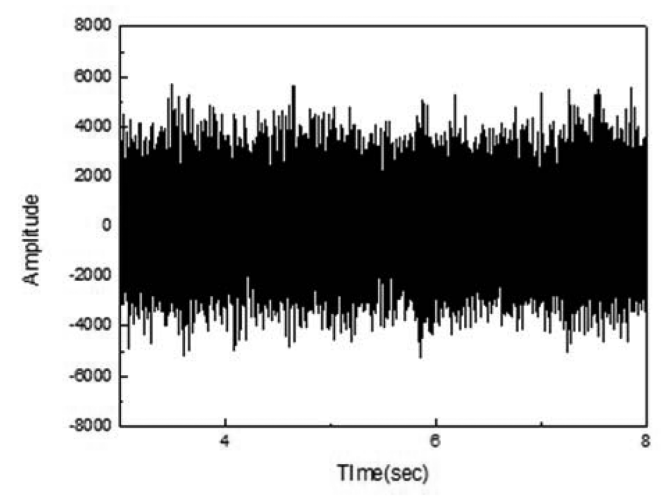

(c) were recorded. The grinded powder particle size according to the rotational speeds of 160,320 , and $352 \mathrm{rpm}$ and times of $1,2,6$, and $24 \mathrm{~h}$, and the acoustics produced by the ball milling process, were analyzed to determine any correlations.

\subsection{Powder Shape and Particle Size Analysis}

The morphology of the starting material and grinded alumina powder were observed using FE-SEM (JSM-6500F, JEOL, Japan). After collecting the starting powder and the

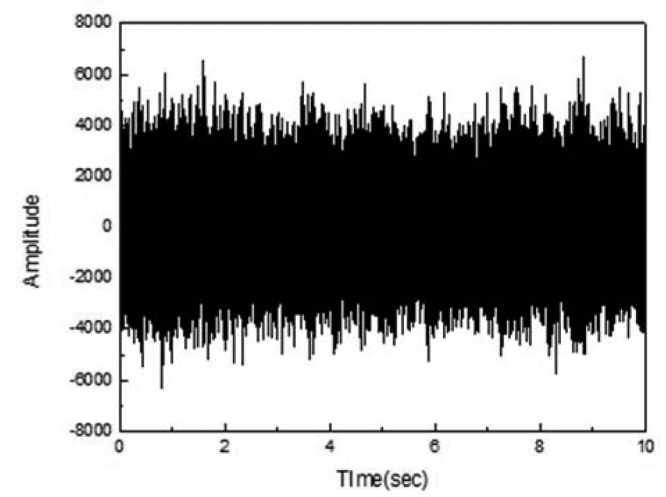

(b)

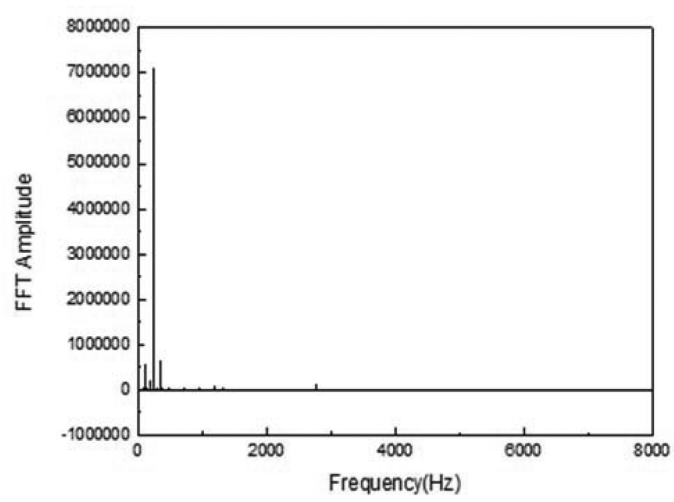

(d)

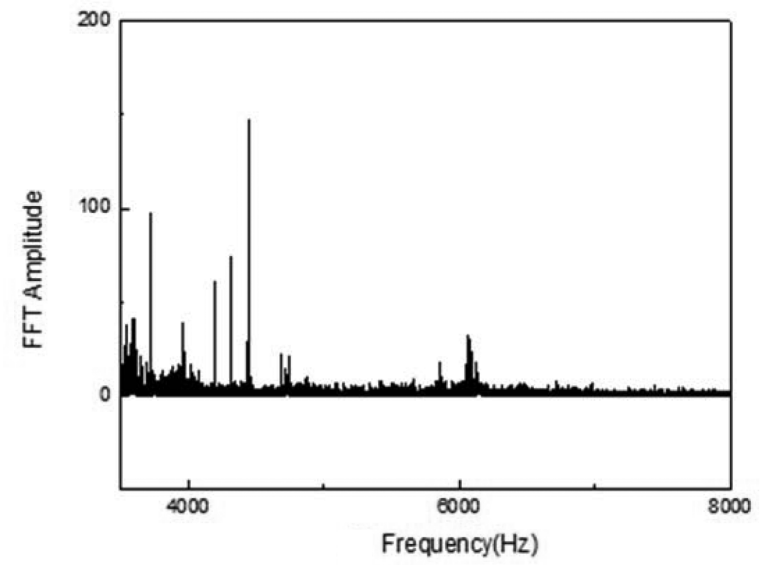

(e)

Fig. 2. Data processing step of acoustic signal for alumina powder ball milling: (a) original signal, (b) passed filter signal, (c) 5 sec selected signal, and the FFT signal at (d) $0 \sim 8,000 \mathrm{~Hz}$ section, (e) 3,500 8,000 $\mathrm{Hz}$ section. 
alumina slurry grinded for each ball milling process parameter, ultrasonic dispersion was carried out for 15 minutes, followed by 10 measurements for particle size analysis using a particle size analyzer (Master Seizer 2000, Malvern Instrument, United Kingdom).

\subsection{Ball Milling Acoustic Signal Acquisition and Data Processing}

The acoustic signal generated by the ball milling was recorded using a voice recorder (WR-01, Doo Won Trade Co., Korea), which was placed within $1 \mathrm{~cm}$ below the ball milling jar; the time domain acoustic data recorded during the ball milling process was uploaded to a personal computer. In order to perform Fourier transform to obtain the frequency domain acoustic data during the ball milling, which was carried out for each rotational speed, acoustic data was acquired for 10 seconds at the $0,1,2,6$, and $24 \mathrm{~h}$ time points. The $0 \mathrm{~h}$ time point refers to the point when the acoustics stabilized, around 30 seconds after the ball milling equipment began operation.

To obtain the frequency domain acoustic data, Fast Fourier Transform (FFT) was performed using the Sigview software (SignalLab Co., USA) for the time domain data of the acoustics generated during the ball milling process; only the effective frequency ranges were selected. In the audio and video fields, FFT transforms the signal data from the time domain to the individual spectrum components in the frequency domain, providing the frequency information of the signal. Fig. 2 shows the processing of the acoustic signal, where (a) shows the initial graph of the time domain data that was recorded during the ball milling process, (b) shows the removal of noise by applying a band pass filter to the recorded sound graph, (c) shows the cutting of the data into 5 second intervals for FFT processing, and (d) shows the time domain acoustic data that were converted to frequency domain data through FFT. Finally, the noise of the frequency range determined to be the inherent mechanical sound of the ball milling device was removed and (e) the acoustic data of only the specific frequency ranges determined to correspond to the powder grinding of the ball milling process were selected.

After this process, the acoustic intensity value was obtained by summing the frequency domain acoustic intensity for the effective range $(3,500 \sim 8,000 \mathrm{~Hz})$ of the 5 second range data selected for FFT from the time domain acoustic data recorded for 10 seconds during the ball milling process for the various rotational speeds and time points.

\section{Results and Discussion}

\subsection{Internal Content Distribution During Ball Mill- ing}

The powder and ball content distribution within the jar during the alumina powder ball milling process for the rotational speed range of $96 \sim 352 \mathrm{rpm}$ was investigated (Fig. 3). For the low rotational speeds of 96 and 128 rpm, the internal content was observed to rotate mainly at positions lower than the center. At $160 \mathrm{rpm}$, the content moved along the wall in the direction opposite to the rotation and the content moved to higher positions as the rotational speed increased.

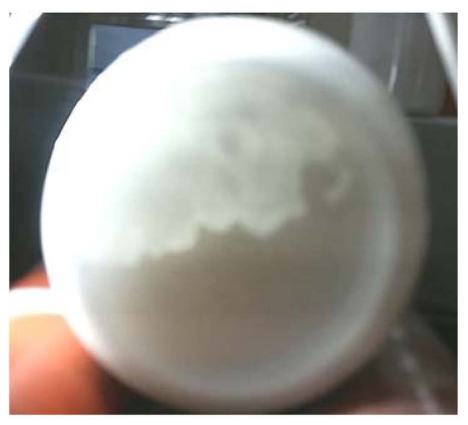

(a)

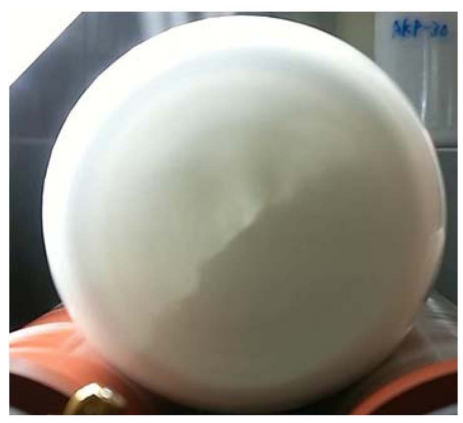

(d)

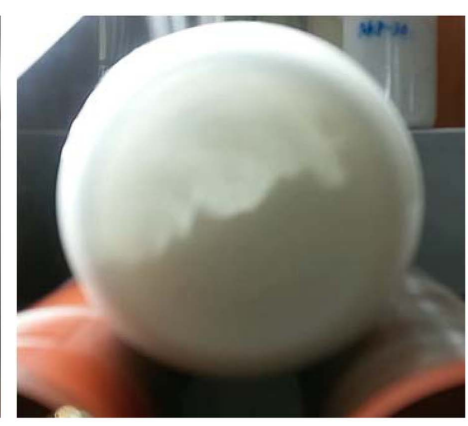

(b)

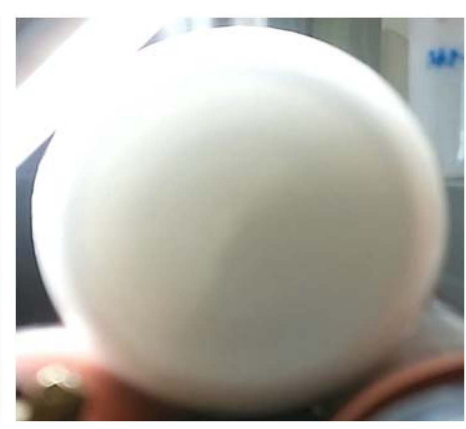

(e)

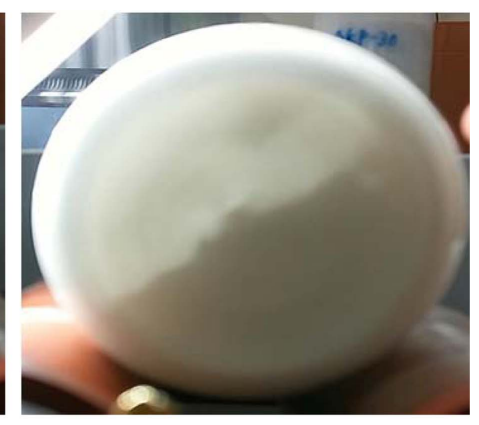

(c)

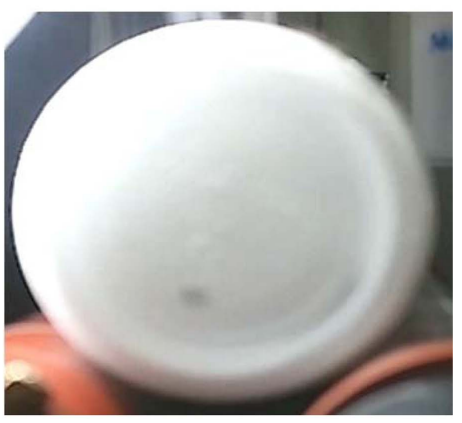

(f)

Fig. 3. Internal distributions of charger (powder and ball) within rotating jar during alumina ball mill process with different rotation speeds: (a) 96, (b) 128, (c) 160, (d) 224, (e) 320, and (f) $352 \mathrm{rpm}$. 
At $320 \mathrm{rpm}$, the internal content rotated as it moved along the internal wall of the jar, while part of the content fell from the upper portion to the lower portion: these were the optimal conditions. For the relatively high rotational speed of $352 \mathrm{rpm}$, a thick layer of content accumulated on the jar internal wall due to the centrifugal force and the jar internals could not be observed in the video recording. A previous study defined the critical rotational speed as the rotational speed at which the content performs rotational motion at

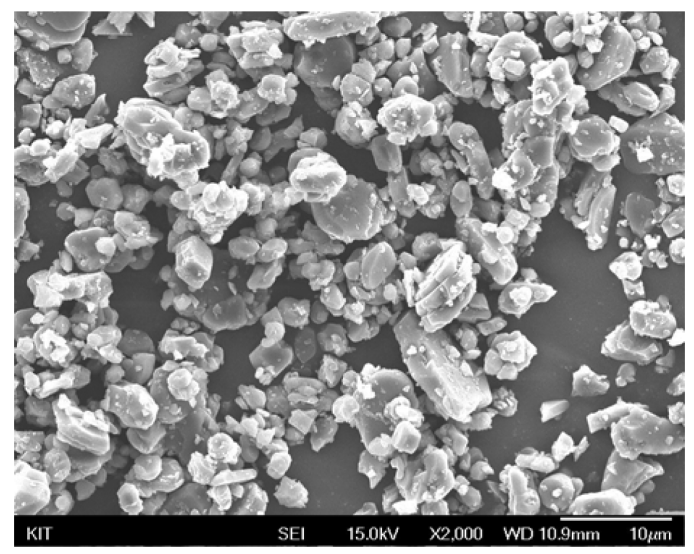

(a)

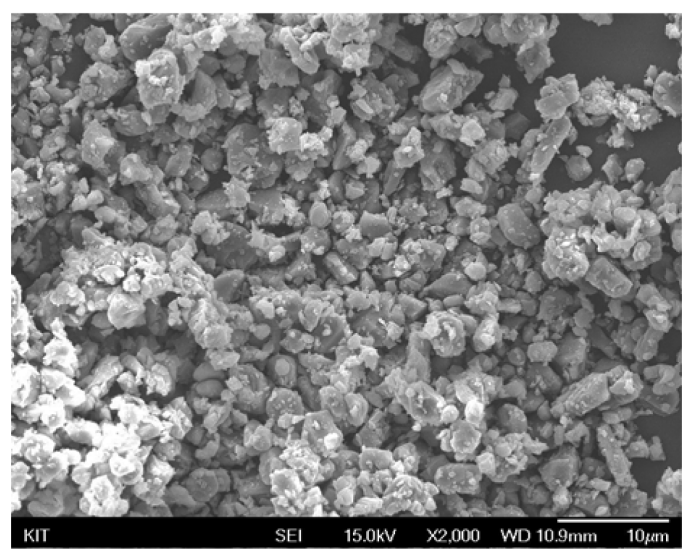

(b)

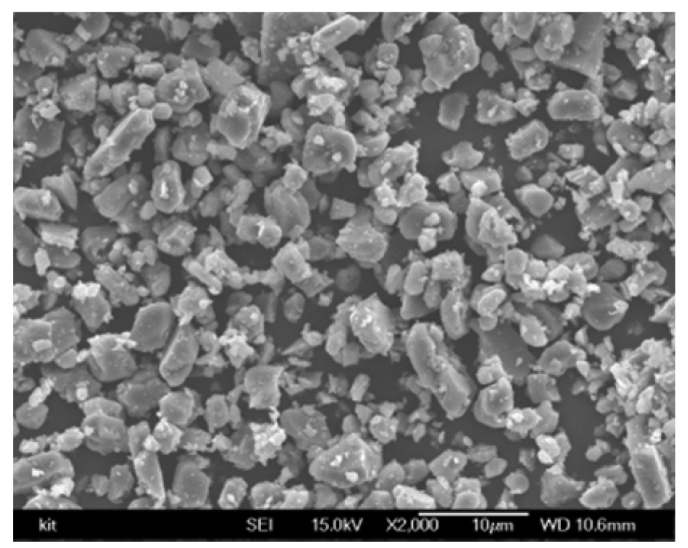

(c)

Fig. 4. Morphology of the ground alumina powder after 24 hrs ball milling with different rotation speeds: (a) 160, (b) 320, and (c) $352 \mathrm{rpm}$. the lower portion of the jar, slanted in the direction opposite to the rotation, all while part of the content is moving due to centrifugal force along the jar internal wall and falling down; that study also analyzed the powder condition. ${ }^{11)}$

Within the scope of the ball milling process in this study, the critical rotational speed was predicted to be $320 \mathrm{rpm}$, with maximum grinding efficiency determined from internal content distribution observation. The ball milling particle size and acoustic intensity were compared for rotational speeds of $160 \mathrm{rpm}$ and $352 \mathrm{rpm}$.

\subsection{Grinded Powder Shape and Particle Size}

3.2.1. Ball Milling Grinded Particle Morphology and Size

The alumina powder grinded for $24 \mathrm{~h}$ at rotational speeds of 160, 320, and $352 \mathrm{rpm}$ were observed through SEM imaging (Fig. 4). The observations revealed that the edges of the irregular polyhedrons of the starting powder experienced abrasion and became spherical. The $320 \mathrm{rpm}$ grinded powder particle size was smaller and had more micropowder than did the powder grinded at 160 and $352 \mathrm{rpm}$. Table 2 shows the size distribution average (vol. weighted mean, $\mathrm{D}[4,3]$ ) and mode for the grinded alumina powder ball milled for $24 \mathrm{~h}$ at rotational speeds of 160, 320, and 352 rpm, compared with the ingredient powder size. The base ingredient, alumina powder, showed an average size of 6.92 $\mu \mathrm{m}$ and mode of $6.25 \mu \mathrm{m}$. In comparison, the ball mill grinded particles were smaller: the particle size average and mode for the rotational speed of $320 \mathrm{rpm}$ were $2.65 \mu \mathrm{m}$ and $3.15 \mu \mathrm{m}$, respectively, showing relatively smaller size compared to that of the powder grinded at the rotational speeds of 160 and $352 \mathrm{rpm}$.

3.2.2. Particle Size Distribution According to Rotational Speed and Time

Figure 5 shows the particle size analysis results for each rotational speed and time. The results show that the particle size decreased with increasing grinding time. The particle size distribution averages for $1,2,6$, and $24 \mathrm{~h}$ at $160 \mathrm{rpm}$ were $5.35,5.24,4.88$, and $4.13 \mu \mathrm{m}$, respectively. The decreasing trend was more significant for the rotational speed of $320 \mathrm{rpm}$, where the size distribution averages were 4.80, $4.69,3.88$, and $2.65 \mu \mathrm{m}$, respectively, for the same time order. On the other hand, this difference decreases for the higher rotational speed of $352 \mathrm{rpm}$.

Table 2. Mean and Mode Sizes of the Starting and 24 h-Milled Alumina Powder as a Function of Rotation Speed

\begin{tabular}{ccc}
\hline $\begin{array}{c}\text { Rotation speed } \\
(\mathrm{rpm})\end{array}$ & \multicolumn{2}{c}{ Milled alumina particle size $(\mu \mathrm{m})$} \\
\cline { 2 - 3 } & mean & mode \\
\hline $0^{*}$ & 6.92 & 6.25 \\
160 & 4.13 & 4.52 \\
320 & 2.65 & 3.15 \\
352 & 3.25 & 3.55 \\
\hline
\end{tabular}

"means the starting powder without ball milling 


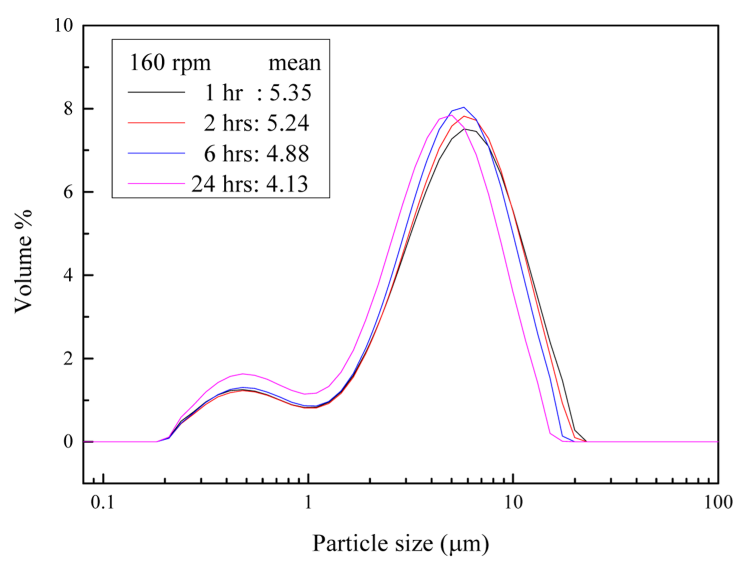

(a)

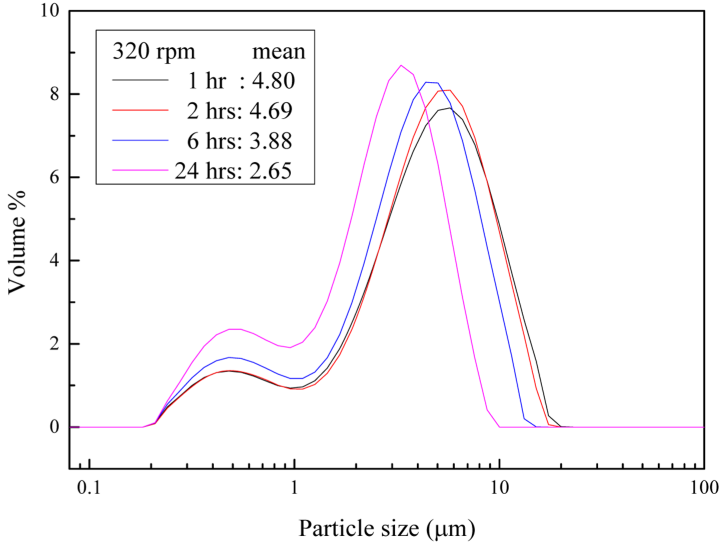

(b)

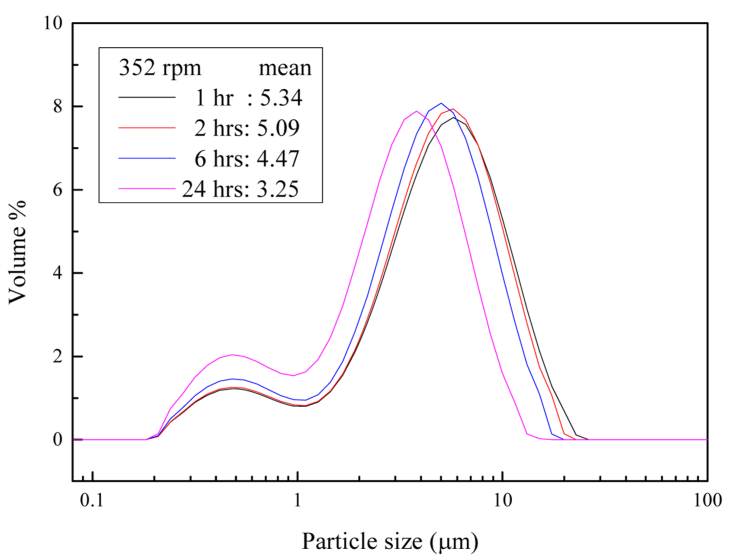

(c)

Fig. 5. Variation of particle size distribution of grained alumina powder with milling time at certain rotation speeds: (a) 160, (b) 320 , and (c) $352 \mathrm{rpm}$.

Figure 6 shows the particle size analysis results according to the rotational speed for each grinding time. For the grinding time of $1 \mathrm{~h}$, the size distribution average decreased to $5.35 \mu \mathrm{m}, 4.80 \mu \mathrm{m}$, and $5.34 \mu \mathrm{m}$ for the rotational speeds of 160, 320, and $352 \mathrm{rpm}$, respectively. The differences between the rotational speeds were not significant; however, the differences in the average and mode decrease for each rotational speed gradually increased as the ball milling process continued to 2 and $6 \mathrm{~h}$. Finally, the size distribution averages for $24 \mathrm{~h}$ grinding were $4.13 \mu \mathrm{m}, 2.65 \mu \mathrm{m}$, and $3.25 \mu \mathrm{m}$ for the rotational speeds of 160,320 , and $352 \mathrm{rpm}$, respectively. Here, the differences were significant. As predicted from the internal content distribution (Fig. 3), the grinding efficiency (\%) values, calculated according to the size decrease rate from the alumina starting material average size to the $24 \mathrm{~h}$ grinded particle average size [(original size - grinded size) / original size] were $40.3 \%(6.92 \rightarrow 4.13 \mu \mathrm{m})$ for 160 $\mathrm{rpm}, 53.0 \%(6.92 \rightarrow 3.25 \mu \mathrm{m})$ for $352 \mathrm{rpm}$, and $61.7 \%(6.92$ $\rightarrow 2.65 \mu \mathrm{m}$ ) for $320 \mathrm{rpm}$, this last of which showed the highest grinding efficiency. Therefore, compared to the slower or faster rotational speeds, it was found that the grinding efficiency was maximized at the rotational speed of $320 \mathrm{rpm}$, considered to be the critical rotational speed.

\subsection{Ball Milling Acoustics}

3.3.1. Ball Milling Acoustic Signal Processing

Figure 7 shows the acoustic intensity graph, expressed in the frequency domain from the Fourier transform of the audio recording for each burden of the same ball milling process at $160 \mathrm{rpm}$. In the figure, (a) corresponds to the ball milling equipment, $(b, c)$ correspond to the jar and the jar filled with water. For the case in which the ball milling was carried out without adding spherical balls or grinding medium, acoustics were observed only for the frequency range of approximately $0 \sim 3,500 \mathrm{~Hz}$; higher frequency acoustics could not be observed. Thus, it was determined that the mechanical acoustics inherent to the ball milling equipment under operation were limited to the frequency range of $0 \sim 1,500 \mathrm{~Hz}$. As can be observed in (d), (e), and (f), the case in which the ball milling was conducted with spherical balls added to the ball milling jar revealed noise inherent to the mechanical equipment for the range of $0 \sim 1,500$ $\mathrm{Hz}$; significant acoustic intensity was observed for the range of $3,500 \sim 8,000 \mathrm{~Hz}$ due to the friction and falling motion of the balls and powder within the jar. Similar to previous studies regarding the sound generated during the ball milling process, ${ }^{13-14)}$ the mechanical sound from the vibration of 


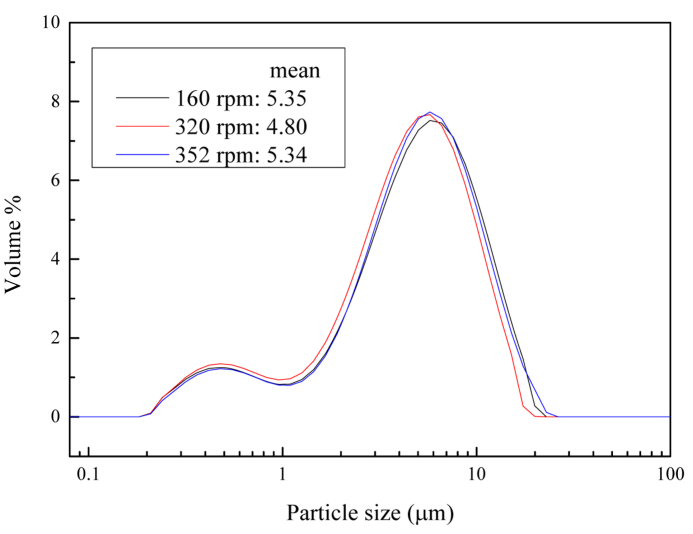

(a)

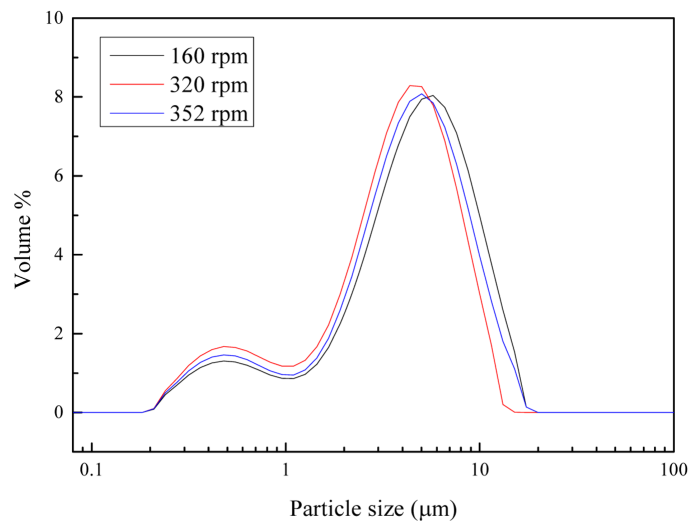

(c)

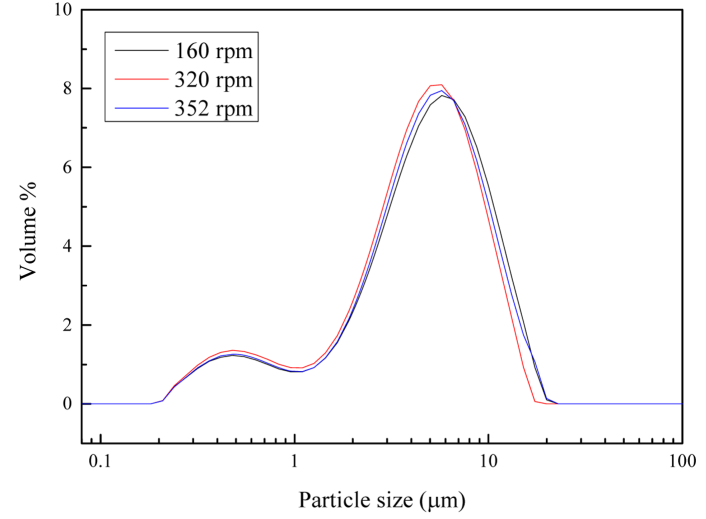

(b)

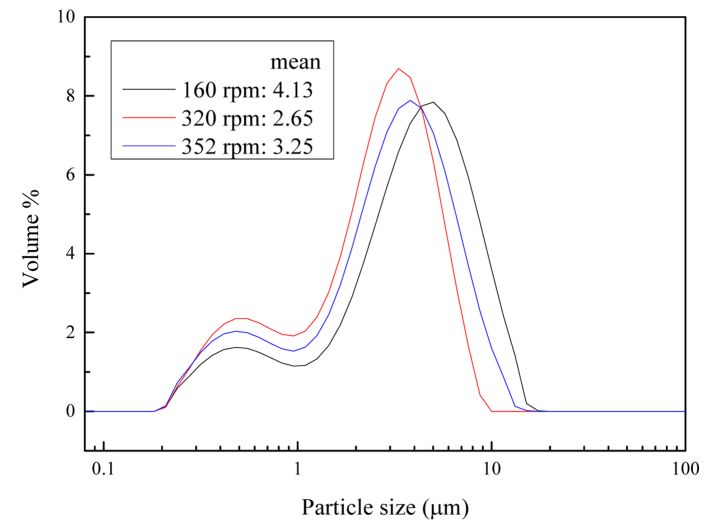

(d)

Fig. 6. Variation of particle size distribution of grained alumina powder with rotation speed at certain milling times: (a) 1, (b) 2, (c) 6 , and (d) $24 \mathrm{~h}$.

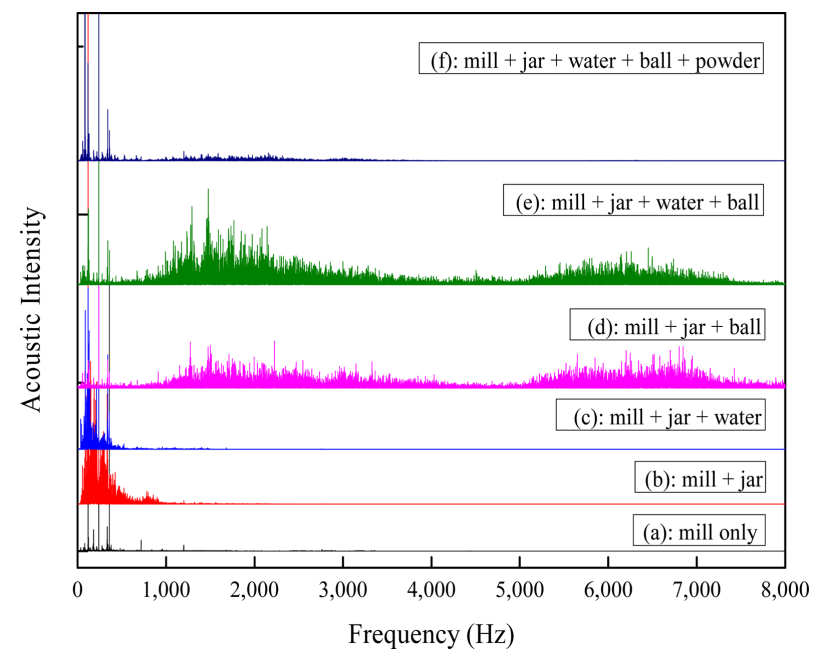

Fig. 7. Acoustic signal graphs as function of frequency obtained from alumina powder ball milling at $160 \mathrm{rpm}$ with different input operation condition.

the equipment itself and the acoustics from the motion of the internal components corresponding to the grinding could be distinguished from their respective frequency ranges. Thus, the following acoustics analysis removed the ball milling equipment mechanical noise in the frequency range of $0 \sim 3,500 \mathrm{~Hz}$ and only the effective acoustics in the range of $3,500 \sim 8,000 \mathrm{~Hz}$ corresponding to the grinding of the ball milling process, were selected.

3.3.2. Acoustics Generated According to Rotational Speed and Time

Figure 8 shows the acoustic graph for the frequency range of 3,500 8,000 Hz, obtained from the Fourier transform for a specific time range (5 seconds) of the acquired acoustics for the rotational speeds of 160 and $320 \mathrm{rpm}$ of ball milling. The acoustic intensity was high for the critical rotational speed $(320 \mathrm{rpm})$ with the greatest grinding efficiency, as determined from the internal content distribution observation and grinded particle size analysis. However, the acoustic intensities for the inadequate rotational speeds (160, 352 rpm) were relatively lower. Fig. 9 shows the acoustic intensity sum of the frequency domain acoustic data extracted for a specific time range (5 seconds) from the acoustic graph acquired for each rotational speed (160, 320, $352 \mathrm{rpm})$ and grinding time $(0,1,2,6,24 \mathrm{~h})$ of the ball milling process. The intensity of the acoustics generated from the ball milling at $160 \mathrm{rpm}$ increased with time to reach a maximum of $30.2 \times 10^{6}$ during the $2 \mathrm{~h}$ ball milling. Afterwards, the acoustic intensity curve plateaued. For the $320 \mathrm{rpm}$ critical rotational speed with maximum grinding efficiency, a high 


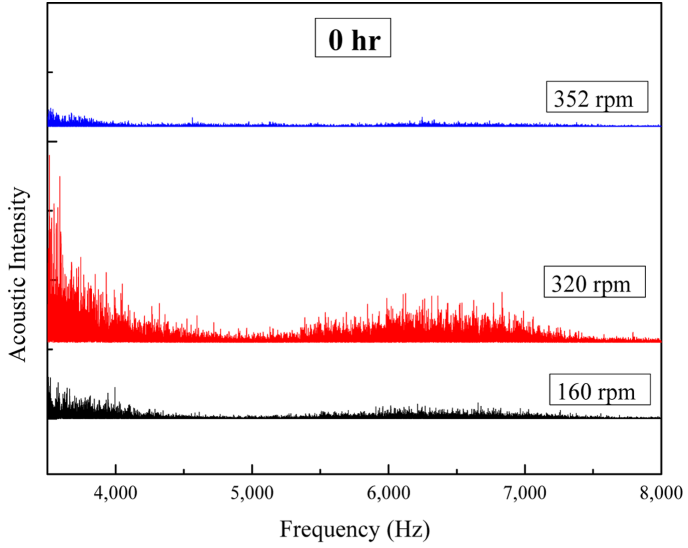

(a)

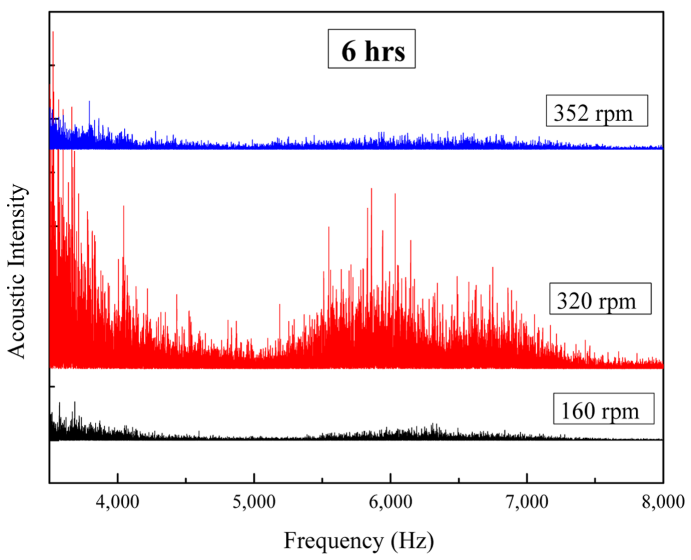

(c)

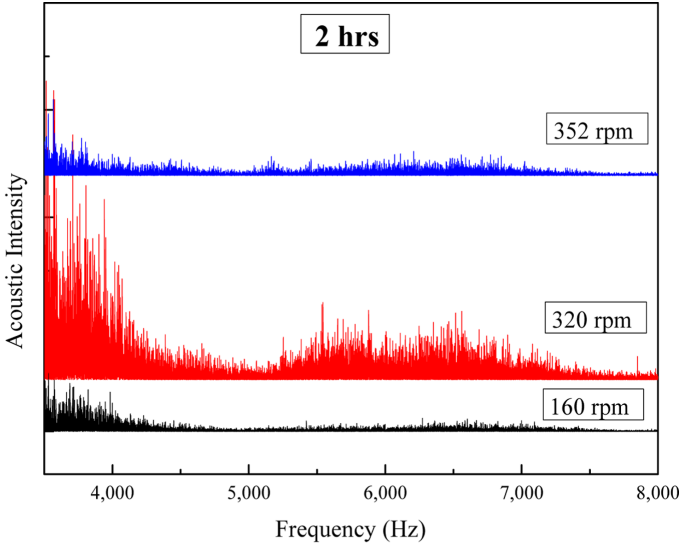

(b)

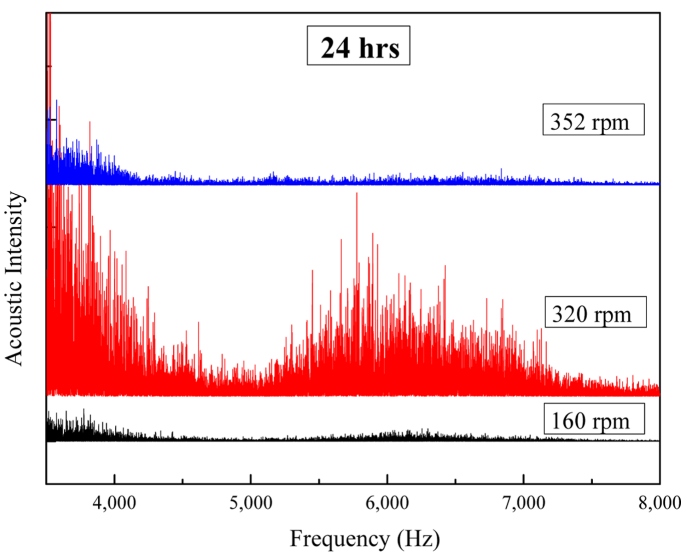

(d)

Fig. 8. Acoustic signal graphs $(3,500 \sim 8,000 \mathrm{~Hz}$ section range) during alumina powder ball milling with different rotation speeds at certain time intervals: (a) 0 , (b) 2 , (c) 6 , and (d) $24 \mathrm{~h}$.

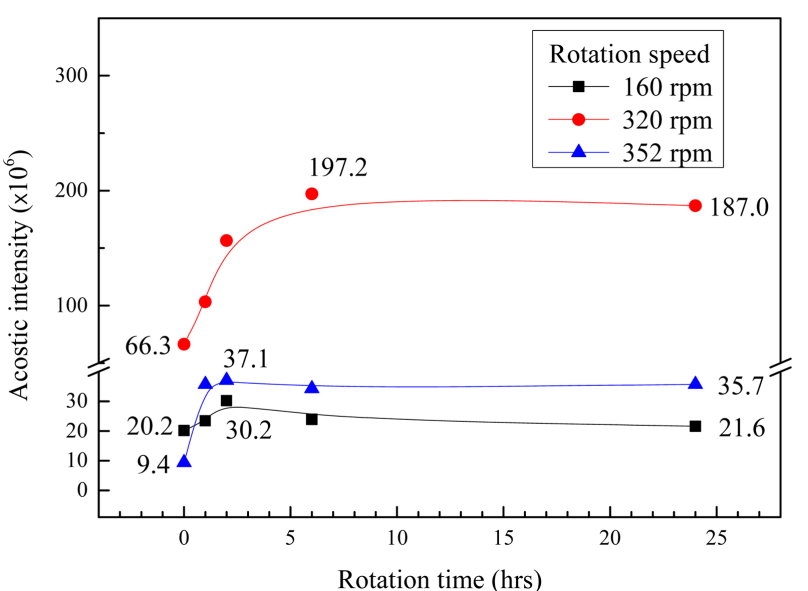

Fig. 9. Variation of acoustic intensity (intensity sum for 5 seconds within $3,500 \sim 8,000 \mathrm{~Hz}$ section) during ball milling with different rotation speeds and time interval.

acoustic intensity of $66.3 \times 10^{6}$ was obtained at the beginning and gradually increased to reach a maximum of 197.2 $\times 10^{6}$ at the $6 \mathrm{~h}$ range and plateau. Overall, the acoustic intensity during the grinding was high. For the rotational speed of $352 \mathrm{rpm}$, which is faster than the critical rotational speed and exhibited a reduced grinding efficiency, the acoustic intensity curve showed trends similar to those for $160 \mathrm{rpm}$ in terms of the maximum acoustic intensity $(37.1 \times$ $\left.10^{6}\right)$ and the time at which the maximum was reached $(2 \mathrm{~h})$.

\subsection{Ball Milling Acoustic Intensity and Particle Size Correlation}

The rotational speed of the ball milling jar was varied to 160,320 , and $352 \mathrm{rpm}$ for the alumina starting powder with an average size of $6.92 \mu \mathrm{m}$ to compare the grinding efficiency (decrease rate of the $24 \mathrm{~h}$ powder particle average size compared to the starting powder) and the acoustics generated for each rotational speed and time $(0,1,2,6$, and 24 h) with the acquired acoustic intensity averages (Fig. 10). The alumina powder grinded for $24 \mathrm{~h}$ at the relatively slower $160 \mathrm{rpm}$ showed a grinding efficiency of $40.3 \%$ and average acoustic intensity of $23.9 \times 10^{6}$, while the grinding efficiency and average acoustic intensity were $53.1 \%$ and $30.4 \times 10^{6}$, respectively, for the relatively faster rotational speed of $352 \mathrm{rpm}$. In comparison, the grinding efficiency and acoustic intensity of the alumina powder particles grinded at $320 \mathrm{rpm}$, considered to be the critical rotational speed, 


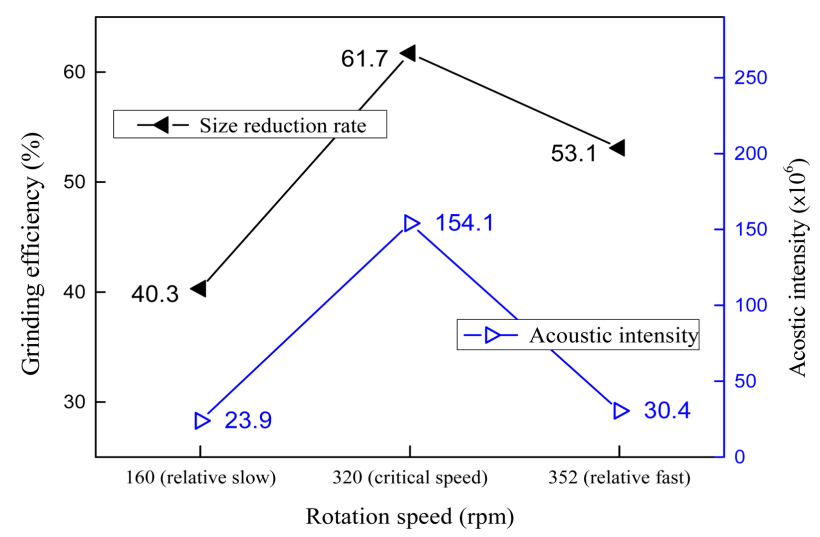

Fig. 10. Relationship between $24 \mathrm{~h}$-grinded mean particle size of alumina powder and acoustic intensity (intensity sum for 5 seconds within 3,500 8,000 $\mathrm{Hz}$ section) by ball milling at different rotation speeds.

were $61.7 \%$ and $154.1 \times 10^{6}$, which were the highest grinding efficiency and acoustic intensity. So, it was observed that the grinding efficiency was high when the generated acoustic intensity was high and the grinding efficiency decreased when the acoustic intensity was lower. The results show that there was a clear correlation between the acoustic intensity generated and the grinding efficiency of the ball milling process.

Ceramic sites and laboratories employ various ball milling jars, ball sizes, ball materials, dispersants, additive types, additive amounts, and rotational speeds. Therefore, significant time and effort are required to identify the conditions for optimal size and formation of the grinded ceramic ingredient powder, as numerous experiments considering a wide range of ball milling conditions need to be conducted, along with analysis of the corresponding grinded particle size distributions. The results of this study reveal that the critical rotational speed condition for optimal grinding can be immediately predicted on site through acoustic intensity measurements.

\section{Conclusions}

The grinded particle size and acoustic intensity for alumina powder were comparatively analyzed considering the variables of the ball milling rotational speed (160, 320, and $352 \mathrm{rpm})$ and grinding time $(0,1,2,6$, and $24 \mathrm{~h})$. The spatial positions of the jar internal content (powder and balls) during the ball milling process were found to concentrate to the bottom when the rotational speed was slow and to the internal wall when the rotational speed was fast, while the content was appropriately distributed to the bottom and internal wall at the appropriate rotational speed. For the critical rotational speed of the alumina powder ball milling, where the rotational speed was optimal, the grinded particle size was smallest; the particle size increased when the rotational speed was slower or faster than the critical rotational speed. Furthermore, the acoustic intensity acquired during ball milling was also highest for the critical rotational speed; the acoustic intensity decreased for slower and faster rotational speeds. Therefore, there was a clear correlation between the acoustic intensity and the grinding efficiency for the ball milling process.

\section{Acknowledgments}

This research was supported by Kumoh National Institute of Technology (2015-104-073).

\section{REFERENCES}

1. L. G. Austin, R. R. Klimpel, and P. T. Luckie, Process Engineering of Size Reduction: Ball Milling; pp. 12-14, 8193, 240-41, Society of Mining Engineers of the AIME, New York, 1984.

2. S. K. Sung, "The Theory of Comminution and the Actual Operation (in Korean)," Cement, 28 58-67 (1969).

3. C.-H. Sim, "The Effect of the Operating Variables on the Batch Ball Milling," Prospect. Ind. Chem., 3 [1] 46-57 (2000).

4. H. Shin, S. Lee, H. S. Jung, and J.-B. Kim, "Effect of Ball Size and Powder Loading on the Milling Efficiency of a Laboratory-Scale Wet Ball Mill," Ceram. Int., 39 [8] 896368 (2013).

5. H. Watanabe, "Critical Rotation Speed for Ball-Milling," Powder Technol., 104 [1] 95-9 (1999).

6. H. Mori, H. Mio, J. Kano, and F. Saito, "Ball Mill Simulation in Wet Grinding Using a Tumbling Mill and Its Correlation to Grinding Rate," Powder Technol., 143-144 23039 (2004).

7. S.-H. Hong and B.-K. Kim, "Effects of Lifter Bars on the Ball Motion and Aluminum Foil Milling in Tumbler Ball Mill," Mater. Lett., 57 [2] 275-79 (2002).

8. N. N. S. Lameck, Effects of Grinding Media Shapes on Ball Mill Performance, pp. 8-39, in M. S. Thesis, University of the Witwatersrand, Johannesburg, 2005.

9. V. Monov, B. Sokolov, and S. Stoenchev, "Grinding in Ball Mills: Modeling and Process Control," Cybern. Inf. Technol., 12 [2] 51-68 (2012).

10. A. S. Kurlov and A. I. Gusev, "Effect of Ball Milling Parameters on the Particle Size in Nanocrystalline Powders," Tech. Phys. Lett., 33 [10] 828-32 (2007).

11. P. W. Cleary, "Predicting Charge Motion, Powder Draw, Segregation and Wear in Ball Mills Using Discrete Element Methods," Miner. Eng., 11 [11] 1061-80 (1998).

12. Hosseini, "Acoustic Emissions Simulation of Tumbling Mills using Charge Dynamics," Miner. Eng., 24 [13] 144047 (2011).

13. J. Tang, L.-J. Zhao, J.-W. Zhou, H. Yue, and T.-Y. Chai, "Experimental Analysis of Wet Mill Load Based on Vibration Signals of Laboratory-Scale Ball Mill Shell," Miner. Eng., 23 [9] 720-30 (2010)

14. S. P. Das, D. P. Das, S. K. Behera, and B. K. Mishra, "Interpretation of Mill Vibration Signal via Wireless Sensing," Miner. Eng., 24 [3-4] 245-51 (2011).

15. B. Behera, B. K. Mishra, and C. V. R. Murty, "Experimen- 
tal Analysis of Charge Dynamics in Tumbling Mills by Vibration Signature Technique," Miner. Eng., 20 [1] 84-91 (2007).

16. F. M. Katubilwa and M. H. Moys, "Effects of Filling Degree and Viscosity of Slurry on Mill Load Orientation," Miner. Eng., 24 [13] 1502-12 (2011).

17. H.-K. Lee, K.-S. Cho, M.-H. Jang, C.-W. Jang, S.-M. Kim, and M.-Y. Kim, "Characteristics of Large Green and Sintered Alumina Ceramics by Filter Pressing," J. Korean Ceram. Soc., 46 [3] 306-12 (2009).

18. K.-S. Cho, J. Kim, S. Y. Lee, J. P. Oh, and S. I. Park,
"Shape Forming of Alumina by Step Pressure-Vacuum Hybrid Slip Casting," Appl. Mech. Mater., 217-219 1899902 (2012).

19. K.-S. Cho, I.-B. Song, and J. Kim, "Homogeneous Shape Forming of Alumina by Pressure-Vacuum Hybrid Slip Casting," J. Korean Ceram. Soc., 49 [6] 592-600 (2012).

20. K.-S. Cho, H.-K. Lee, and B.-J. Woo, "Effect of Step Pressure on Shape Forming of Alumina by Pressure-Vacuum Hybrid Slip Casting," J. Korean Ceram. Soc., 50 [2] 14248 (2013). 